\title{
Clinical Diagnosis, Biochemical Findings, Genetics and Incidence of
} Zellweger Syndrome

\author{
Muhittin Celik,,$^{, *}$ Mehmet Sah Ipek, ${ }^{2}$ Nezir Ozgun, ${ }^{3}$ Osman Akdeniz, ${ }^{4}$ Heybet Tuzun, ${ }^{1}$ and Ali Bulbul ${ }^{5}$ \\ ${ }^{1}$ M.D. Diyarbakir Children's Hospital, Clinic of Neonatology, Diyarbakir, Turkey \\ ${ }^{2}$ M.D. Division of Neonatology, Department of Pediatrics, Memorial Hospital, Diyarbakir, Turkey \\ ${ }^{3}$ M.D. Diyarbakir Children's Hospital, Clinic of Pediatric Neurology, Diyarbakir, Turkey \\ ${ }^{4}$ M.D. Diyarbakir Children's Hospital, Clinic of Pediatric Cardiology, Diyarbakir, Turkey \\ ${ }^{5}$ Ass. Prof. Sisli Hamideyi Etfal Research Hospital, Clinic of Neonatology, Istanbul, Turkey \\ "Corresponding author: Dr. Muhittin Celik, Division of Neonatology, Department of Pediatrics, Diyarbakir Children's Hospital, Diyarbakir, Turkey. Tel: +90-4122245751-1506, Fax: \\ +90-4122287758, E-mail: mehdincelik@hotmail.com
}

Received 2017 July 27; Revised 2017 October 06; Accepted 2017 November 16.

\begin{abstract}
Objectives: To analyze the clinical and laboratory data of neonates diagnosed with Zellweger syndrome and to estimate the incidence of the syndrome.

Methods: The databases of four institutions that admitted newborns diagnosed with Zellweger syndrome to intensive care units between January 2013 and December 2016 were examined.

Results: A total of 105,887 live babies were born in the four centers during the study period. Seven were diagnosed with Zellweger syndrome; the incidence was thus 1/15,126. Birth weights were 2,200 - 3,300 g. Six cases were born to consanguineous parents. Dysmorphic findings, respiratory failure, and hypotonia were evident in all patients. Hepatomegaly was apparent in four cases, congenital cardiac defects in four, characteristic cerebral magnetic resonance imaging findings in four, renal cysts in five, hepatic cysts in three, and splenomegaly in one. PEX1 mutations were identified in three patients, and one mutation was novel.

Conclusions: The incidences of Zellweger syndrome and cardiac disease were higher than reported previously, being (to the best of our knowledge) among the highest reported worldwide. This is the first time that such incidences have been calculated in Turkey. The syndrome is more common in regions where consanguineous marriage rates are higher.
\end{abstract}

Keywords: Cardiac Disease, Neonatal Period, Zellweger Syndrome

\section{Background}

Peroxisome biogenesis disorders (PBDs; OMIM \#601539) constitute a group of neurodegenerative disorders characterized by defective peroxisome assembly and dysfunction of multiple peroxisomal enzymes ( 1 , 2). PBDs are inherited in an autosomal recessive manner (2). PBDs are composed of two clinically distinct subtypes, Rhizomelic Chondrodysplasia Punctata Type 1 (RCDP; OMIM \#215100) and Zellweger Spectrum Disorder (ZSD), which together account for about $80 \%$ of all PBD patients. ZSD includes Zellweger syndrome (ZS; OMIM \#214100), neonatal adrenoleukodystrophy (NALD; OMIM \#202370), infantile Refsum disease (IRD; OMIM \#266510), and Heimler syndrome $(2,3)$.

Infants with ZS typically present in the neonatal period with profound muscular hypotonia, poor feeding behavior, frequent seizures, jaundice, hepatic dysfunction, bone abnormalities, and specific craniofacial dysmorphism (4, $5)$. The infants have poor prognoses and usually die within the first year of life $(4,6)$.

Zellweger spectrum disorder is caused by mutations in any of 14 currently known PEX genes, which encode proteins termed peroxins that are involved in either peroxisome formation, peroxisomal protein importation, or both (5-7). Consequently, mutations in PEX genes cause deficiencies in peroxisome numbers or function, or mosaic patterns $(5,6,8)$. Peroxisomes are present in all cells except mature erythrocytes and are particularly common in renal and hepatic cells $(9,10)$. Peroxisomes are involved in many anabolic and catabolic processes, including the $\beta$ oxidation of very-long-chain fatty acids (VLCFAs); the synthesis of other lipids, such as plasmalogens; and bile-acid metabolism $(9,11)$.

A clinical diagnosis of ZS can be definitively confirmed by laboratory assays of readily accessible materials such as blood and urine. Measurement of plasma VLCFA levels is commonly the first test performed (5). In cases of high clinical suspicion of $\mathrm{ZS}$, the fact that the peroxisomal param- 
eters of blood and urine are initially normal does not exclude ZS, and further testing (including fibroblast culture and/or molecular analyses) should be considered $(12,13)$. The syndrome affects liver function and triggers cardiac anomalies, but the extent of liver dysfunction over time, the incidence of cardiac anomalies, and the overall incidence of the syndrome remain unknown in Turkey.

Although the incidence of ZS differs by region, the overall incidence is estimated to be 1 in 50,000-100,000 births $(5,14)$. We reviewed seven patients diagnosed with ZS in four centers in Diyarbakir, Turkey, over the past 4 years and estimated the incidence of ZS.

\section{Methods}

We retrospectively reviewed the files of eight patients admitted to neonatal intensive care units with Zellweger syndrome between January 2013 and December 2016. One patient who did not fulfilled our enrollment criteria was excluded. Birth records, birth weights, parental genetic status, findings on physical examination, complaints, laboratory blood data, radiological information, echocardiographic data, and prognoses were retrieved for all seven cases.

\subsection{Diagnostic Criteria for Zellweger Syndrome}

1. Classic phenotypic features (high forehead, large anterior fontanelle, markedly separated cranial sutures, hypoplastic supraorbital ridges, upward-slanting eyes, epicanthal folds, a low and broad nasal bridge, a highly arched palate, and deformed ear lobes)

2. Supportive clinical findings (severe hypotonia and hepatomegaly) and radiological findings (renal, cerebral, and/or liver cyst[s])

3. VLCFA analysis: level of C26:0 fatty acids and C26/C22 and $\mathrm{C} 24 / \mathrm{C} 22$ ratios within the diagnostic ranges for ZS (1.8 - $8.1 \mu \mathrm{mol} / \mathrm{L}, 0.069$ - 0.450, and 0.90 - 2.52, respectively)

4. A PEX mutation if mutational analyses were performed

During the study period, a total of 105,887 live births were recorded in the four centers, and the incidence of ZS was 1 case per 12,191 live births.

Ethics approval was not required because the study was retrospective in nature (based on medical records); the guidelines of our ministry of health exempt such studies from review.

\section{Results}

Demographic, clinical, and radiographic features and patient outcomes are shown in Table 1 . Six sets of parents were consanguineous. Six babies were born at term, and one was born at near term. Three were born with stained meconium and two required intubation in the delivery room. All seven were admitted to neonatal intensive care units with respiratory distress, poor feeding ability, hypotonia, and/or convulsions.

In addition to hypotonia, typical craniofacial features, including epicanthal folds, a high forehead, a broad nasal bridge, hypoplastic supraorbital ridges, and an enlarged anterior fontanelle, were present in all patients. Five patients presented with hepatomegaly, and one also exhibited splenomegaly. At admission, biochemical and hematological data were unremarkable except for a mildly elevated transaminase level in one patient. The plasma levels of VLCFAs were markedly increased in all patients: the C26:0 level ranged from 5.14 to $14.05 \mu \mathrm{mol} / \mathrm{L}$, the C24:C22 ratio ranged from 1.47 to 1.82 , and the $\mathrm{C} 26: \mathrm{C} 22$ ratio ranged from 0.24 to 0.50 (Table 1). Four patients exhibited abnormalities on echocardiograph, including atrioventricular and atrial septal defects and tetralogy of Fallot. Abdominal ultrasonography detected renal cysts in six patients and hepatic cysts in three patients. Brain magnetic resonance imaging revealed cerebral germinolytic cysts in three patients; bilateral ventricular dilation and agenesis of the corpus callosum in one patient; and variable gyral abnormality, enlarged lateral ventricles, and hypomyelination in all seven patients.

Molecular analysis of PEX genes was performed in only three patients, who were found to carry PEX1 mutations. Their parents were carriers of the same mutation, and one parent had a history of affected siblings. Patients 1 and 7 had a PEX1; c.2097insT (homozygous) mutation, whereas patient 4 had a PEX1; c.1359 + 5G > A (homozygous)/PEX6; c.2905C $>\mathrm{T}$ (heterozygous) mutation.

\subsection{Follow-Up and Outcomes}

Convulsions developed soon after delivery in two patients but only after 1 month in one. Two patients did not exhibit any clinically observed convulsions prior to death. All patients received supportive symptom-based interventions. Liver function tests (including transaminase levels) become abnormal in the first month of life among all surviving patients and continued to deteriorate. Six patients required late intubation and then died. One patient still survives (at 6 months of age).

\subsection{Estimated Incidence}

During the study period, a total of 105,887 live births were recorded in four hospitals in Diyarbakir. The estimated ZS incidence was 1/15,126 live births (95\% confidence interval 1/9,500 - 1/58,727). 
Table 1. Demographic, Clinical, and Radiographic Features of the Patients

\begin{tabular}{|c|c|c|c|c|c|c|c|}
\hline Features & Patient 1 & Patient 2 & Patient 3 & Patient 4 & Patient 5 & Patient 6 & Patient 7 \\
\hline Gender & $\mathrm{F}$ & M & $\mathrm{F}$ & M & $\mathrm{F}$ & M & $\mathrm{F}$ \\
\hline Parental consanguinity & - & + & + & + & + & + & + \\
\hline Convulsion & $t^{\mathrm{a}}$ & + & - & + & - & $+^{\mathrm{a}}$ & - \\
\hline Hypotonia & + & + & + & + & + & + & + \\
\hline Typical face & + & + & + & + & + & + & + \\
\hline Ocular abnormality & $++^{\mathrm{b}}$ & $+^{\mathrm{c}}$ & - & - & NA & $t^{\mathrm{b}}$ & - \\
\hline Hepatomegaly & + & + & + & - & - & + & + \\
\hline Splenomegaly & + & - & - & - & - & - & - \\
\hline Cerebral cysts & - & + & + & $+^{\mathrm{d}}$ & - & - & - \\
\hline Abnormal cortical gyral pattern & + & + & \pm & + & \pm & + & - \\
\hline Renal cysts & + & + & + & + & + & - & + \\
\hline Hepatic cysts & + & + & - & + & - & - & - \\
\hline Cardiac defects & $+^{e}$ & - & $+^{e}$ & $++^{\mathrm{f}}$ & - & $+^{g}$ & - \\
\hline Elevated transaminases (at admission) & - & - & - & + & - & - & - \\
\hline Elevated transaminases (at the first month) & + & + & + & + & NA & + & + \\
\hline $\mathrm{C26}, \mu \mathrm{mol} / \mathrm{L}$ (Diagnostic range:1.8 - 8.1) & 14,05 & 5,14 & 7,82 & 9,37 & 9,12 & 6,62 & 9,16 \\
\hline C26:C22 (Diagnostic range:0.069 - 0.45) & 0,38 & 0,48 & 0,50 & 0,43 & 0,31 & 0,24 & 0,29 \\
\hline C24:C22 (Diagnostic range:0.90 - 2.52) & 1,75 & 1,82 & 1,50 & 1,71 & 1,61 & 1,47 & 1,74 \\
\hline Mutation & PEX $1^{\mathrm{h}}$ & NA & NA & PEX1 $1^{\mathrm{i}}$ & NA & NA & PEXi ${ }^{h}$ \\
\hline Age at death, $d$ & 80 & 147 & 46 & 44 & 17 & 93 & Live \\
\hline
\end{tabular}

Abbreviations: F, female; $\mathrm{M}$, male; NA, not available.

${ }^{a}$ Began soon after birth.

${ }^{\mathrm{b}}$ Abnormal retinal pigmentation.

${ }^{\mathrm{c}}$ Cataract.

${ }^{\mathrm{d}}$ Agenesis of corpus callosum, ventricle enlargement.

${ }^{\mathrm{e}}$ Ventricular septal defect and atrial septal defect.

${ }^{\mathrm{f}}$ Atrial septal defect.

${ }^{\mathrm{g}}$ Tetralogy of Fallot.

${ }^{h}$ PEX1; c.2097insT (Homozygous)

${ }^{\mathrm{i}}$ PEX1; c.1359 + 5G > A (Homozygous), PEX6; c.2905C > T (Heterozygous).

\section{Discussion}

Here we report on six newborns with the classic clinical, neuroradiological, and laboratory features of ZS. The estimated incidence of ZS in our local region is higher than in previous reports $(9,11,12)$. ZS was initially described in several members of a single family with multiple congenital anomalies involving the brain, liver, and kidneys; the cited authors described the phenotype as a "cerebrohepatorenal" syndrome (13-15). Affected patients present soon after birth with profound hypotonia, a distinctive facial appearance, seizures, renal cysts, liver cysts associated with hepatic dysfunction, and an inability to feed. Retinopathy and deafness commence in the first months of life, followed by severe brain dysfunction associated with a disorder of neuronal migration.
The liver disease of ZS is typical, manifesting as hepatomegaly, conjugated hyperbilirubinemia, and abnormal liver function test results (5). In some patients, liver disease can progress to splenomegaly, cirrhosis, and portal hypertension (15). The progression of liver disease is rather variable $(5,15)$. In this series, only one patient exhibited elevated transaminase levels on admission, but toward the end of the first month, most patients had developed hepatic involvement. Cardiac defects, including septal defects and aortic abnormalities, have also been reported in ZS patients $(5,14,16)$. In the present series, four of the six patients had cardiac anomalies.

Based on clinically and biochemically diagnosed ZS cases over a 3-year period, we estimated the incidence of ZS in the province of Diyarbakir, Turkey, to be 1/15,126 live 
births. This is higher than its worldwide prevalence (9, $14)$, although the confidence interval is large (1/9,500 $1 / 58,727)$. Similar high incidences have been reported in the Saguenay-Lac-St-Jean region (SLSJ) of Quebec, Canada $(1 / 12,191)(17)$, in the Okinawa Islands of Japan $(1 / 30,000)(12)$, and among Karaites in Israel $(1 / 25,000)(18)$. This may reflect community isolation or a founder effect (17), both of which reduce genetic diversity. However, we suggest that such explanations are not relevant here; our region is cosmopolitan, containing persons of many different ethnicities. The rate of parental consanguinity in eastern Turkey may reach $20 \%$ (19), which explains the high observed incidence of ZS. Our region lacks a comprehensive genetics service. In addition, as hypotonia and respiratory failure are associated with other perinatal problems (such as perinatal asphyxia), it is likely that some cases were missed.

The underlying mechanism of ZS is poorly understood, but it has long been considered that VLCFA accumulation, loss of plasmalogens, and deficiency in materials synthesized by peroxisomes may contribute to the observed brain pathogenesis $(6,15,20)$. In the absence of a distinct clinical presentation, cerebral magnetic resonance imaging identifies several rather characteristic features in patients with peroxisomal disorders and is often a valuable tool in terms of diagnostic workup (21, 22). ZS patients may exhibit neocortical dysplasia (particularly a characteristic perisylvian polymicrogyria), a generalized decrease in white matter volume, delayed myelination, bilateral ventricular dilation, and germinolytic cysts $(5,21,22)$. The cysts have a characteristic appearance and can be useful for confirming suspected ZS, as they are easily detected by transfontanelle sonography and magnetic resonance imaging (22). In our present series, a variety of features consistent with ZS were evident in all patients.

PEX1 (OMIM \#602136) and PEX6 (OMIM \#601498) mutations are the most common causes of ZS, associated with the full continuum of clinical phenotypes $(10,23)$. However, a general relationship appears to exist among genotype, cellular phenotype (i.e., importation of peroxisomal matrix proteins), and clinical phenotype (24). No comprehensive PEX molecular genetic testing is available in Turkey; we thus lack data on phenotype/genotype correlations. Unfortunately, we could not perform molecular genetic testing on all patients in our present series because of limited laboratory facilities. However, all three patients who were examined had PEX1 mutations, of which one was new. A study from Saudi Arabia found that a missense mutation in the PEX5 gene was the most common mutation among ZS patients in that country (25). We suggest that the high rate of parental consanguinity in our region allows a founder mutation to persist. However, the nature of the pathogenic alleles in our population requires further study.

In instances of clinical suspicion of ZS associated with abnormal VLCFA levels, subsequent PEX mutational analysis should ideally be performed to confirm the diagnosis $(5,11,21)$. Complementation studies (including fibroblast tests) usefully distinguish peroxisome assembly defects from deficiencies in single peroxisomal enzymes (particularly D-bifunctional protein; patients thus affected rather closely resemble ZS patients) and identify familial pathogenic mutations that raise a need for genetic counseling $(5,7,11)$.

Current ZS treatments are supportive, focusing on amelioration of seizures and liver dysfunction, adequate calorie intake, provision of hearing aids, ophthalmological interventions, and attention to other developmental needs $(5,11)$. As in our case series, ZS is always fatal, but with optimal care and a favorable genetic/phenotypic profile, some patients may live for up to 2 years (14). ZS patients usually die during the first year of life secondary to progressive apnea or respiratory compromise caused by infection $(5,7,11)$.

In conclusion, we report an unusually high incidence of ZS in the province of Diyarbakir that is very likely attributable to parental consanguinity. In addition, the frequency of cardiac abnormalities in patients with ZS is higher than we expected. We found a new causative mutation. The pool of pathogenic alleles in our region requires further study.

\section{References}

1. Sun Y, Wang L, Wei X, Zhu Q, Yang Y, Lan Z, et al. Analysis of a Chinese pedigree with Zellweger syndrome reveals a novel PEX1 mutation by next-generation sequencing. Clin Chim Acta. 2013;417:57-61. doi:10.1016/j.cca.2012.12.005. [PubMed: 23247051].

2. Fujiki Y, Yagita Y, Matsuzaki T. Peroxisome biogenesis disorders: molecular basis for impaired peroxisomal membrane assembly: in metabolic functions and biogenesis of peroxisomes in health and disease. Biochim Biophys Acta. 2012;1822(9):1337-42. doi: 10.1016/j.bbadis.2012.06.004. [PubMed: 22705440].

3. Ratbi I, Falkenberg KD, Sommen M, Al-Sheqaih N, Guaoua S, Vandeweyer G, et al. Heimler Syndrome Is Caused by Hypomorphic Mutations in the Peroxisome-Biogenesis Genes PEX1 and PEX6. Am J Hum Genet. 2015;97(4):535-45. doi: 10.1016/j.ajhg.2015.08.011. [PubMed: 26387595].

4. Salpietro V, Phadke R, Saggar A, Hargreaves IP, Yates R, Fokoloros C, et al. Zellweger syndrome and secondary mitochondrial myopathy. Eur J Pediatr. 2015;174(4):557-63. doi: 10.1007/s00431-014-2431-2. [PubMed: 25287621].

5. Klouwer FC, Berendse K, Ferdinandusse S, Wanders RJ, Engelen M, PollThe BT. Zellweger spectrum disorders: clinical overview and management approach. Orphanet JRare Dis. 2015;10:151. doi:10.1186/s13023-0150368-9. [PubMed: 26627182].

6. Crane DI. Revisiting the neuropathogenesis of Zellweger syndrome. Neurochem Int. 2014;69:1-8. doi: 10.1016/j.neuint.2014.02.007. [PubMed: 24607700]. 
7. Braverman NE, D'Agostino MD, Maclean GE. Peroxisome biogenesis disorders: Biological, clinical and pathophysiological perspectives. Dev Disabil Res Rev. 2013;17(3):187-96. doi: 10.1002/ddrr.1113. [PubMed: 23798008].

8. Gootjes J, Schmohl F, Mooijer PA, Dekker C, Mandel H, Topcu M, et al. Identification of the molecular defect in patients with peroxisomal mosaicism using a novel method involving culturing of cells at 40 degrees C: implications for other inborn errors of metabolism. Hum Mutat. 2004;24(2):130-9. doi: 10.1002/humu.20062. [PubMed:15241794].

9. Schutgens RB, Heymans HS, Wanders RJ, van den Bosch H, Tager JM. Peroxisomal disorders: a newly recognised group of genetic diseases. Eur J Pediatr. 1986;144(5):430-40. [PubMed: 3514227].

10. Wanders RJ, Waterham HR. Biochemistry of mammalian peroxisomes revisited. Annu Rev Biochem. 2006;75:295-332. doi: 10.1146/annurev.biochem.74.082803.133329. [PubMed: 16756494].

11. Reuber BE, Germain-Lee E, Collins CS, Morrell JC, Ameritunga R, Moser HW, et al. Mutations in PEX1 are the most common cause of peroxisome biogenesis disorders. Nat Genet. 1997;17(4):445-8. doi: 10.1038/ng1297-445. [PubMed: 9398847].

12. Zeharia A, Ebberink MS, Wanders RJ, Waterham HR, Gutman A, Nissenkorn A, et al. A novel PEX12 mutation identified as the cause of a peroxisomal biogenesis disorder with mild clinical phenotype, mild biochemical abnormalities in fibroblasts and a mosaic catalase immunofluorescence pattern, even at 40 degrees C. J Hum Genet. 2007;52(7):599-606. doi: 10.1007/s10038-007-0157-y. [PubMed: 17534573].

13. Steinberg SJ, Snowden A, Braverman NE, Chen L, Watkins PA, Clayton PT, et al. A PEX10 defect in a patient with no detectable defect in peroxisome assembly or metabolism in cultured fibroblasts. $J$ Inherit Metab Dis. 2009;32(1):109-19. doi: 10.1007/s10545-008-0969-8. [PubMed: 19127411].

14. Gould SJ, Raymond GV, Valle D. The peroxisome biogenesis disorders. In: Scriver CR, Sly WS, Valle D, editors. The Metabolic and MolecularBases of Inherited Disease. New York: McGraw-Hill; 2001. p. 3181-18.

15. Steinberg SJ, Dodt G, Raymond GV, Braverman NE, Moser AB, Moser HW. Peroxisome biogenesis disorders. Biochim Biophys Acta. 2006;1763(12):1733-48. doi: 10.1016/j.bbamcr.2006.09.010. [PubMed: 17055079].

16. Shimozawa N, Nagase T, Takemoto Y, Ohura T, Suzuki Y, Kondo N.
Genetic heterogeneity of peroxisome biogenesis disorders among Japanese patients: evidence for a founder haplotype for the most common PEX10 gene mutation. Am J Med Genet A. 2003;120A(1):40-3. doi: 10.1002/ajmg.a.20030. [PubMed: 12794690].

17. Bowen P, Lee CS, Zellweger H, Lindenberg R. A Familial Syndrome of Multiple Congenital Defects. Bull Johns Hopkins Hosp. 1964;114:402-14. [PubMed: 14169466].

18. Lee PR, Raymond GV. Child neurology: Zellweger syndrome. Neurology. 2013;80(20):e207-10. doi: 10.1212/WNL.ob013e3182929f8e. [PubMed: 23671347].

19. Steinberg S, Chen L, Wei L, Moser A, Moser H, Cutting G, et al. The PEX Gene Screen: molecular diagnosis of peroxisome biogenesis disorders in the Zellweger syndrome spectrum. Mol Genet Metab. 2004;83(3):252-63. doi: 10.1016/j.ymgme.2004.08.008. [PubMed: 15542397].

20. Wanders RJ. Metabolic and molecular basis of peroxisomal disorders: a review. Am J Med Genet A. 2004;126A(4):355-75. doi: 10.1002/ajmg.a.20661. [PubMed: 15098234].

21. Dursun A, Gucer S, Ebberink MS, Yigit S, Wanders RJ, Waterham HR. Zellweger syndrome with unusual findings: non-immune hydrops fetalis, dermal erythropoiesis and hypoplastic toe nails. J Inherit Metab Dis. 2009;32 Suppl 1:S345-8. doi:10.1007/s10545-009-90100. [PubMed: 20033294].

22. Levesque S, Morin C, Guay SP, Villeneuve J, Marquis P, Yik WY, et al. A founder mutation in the PEX6 gene is responsible for increased incidence of Zellweger syndrome in a French Canadian population. BMC Med Genet. 2012;13:72. doi:10.1186/1471-2350-13-72. [PubMed: 22894767].

23. Zung A, Mogilner BM, Nissani R, Appelman Z, Gelman de Kohan Z. Occurrence of cerebrohepatorenal (Zellweger) syndrome in the Karaite community in Israel: a genetic hypothesis. Isr J Med Sci. 1990;26(10):570-2. [PubMed: 2249933].

24. Kaplan S, Pinar G, Kaplan B, Aslantekin F, Karabulut E, Ayar B, et al. The Prevalence of Consanguineous Marriages and Affecting Factors in Turkey: A National Survey. J Biosoc Sci. 2016;48(5):616-30. doi: 10.1017/S0021932016000055. [PubMed: 26892044].

25. Powers JM, Tummons RC, Caviness VJ, Moser AB, Moser HW. Structural and chemical alterations in the cerebral maldevelopment of fetal cerebro-hepato-renal (Zellweger) syndrome. J Neuropathol Exp Neurol.1989;48(3):270-89. [PubMed: 2703857]. 